\title{
Joint gateway selection, transmission slot assignment, routing and power control for wireless mesh networks ${ }^{2 /}$
}

\author{
Kagan Gokbayrak ${ }^{\mathrm{a}, *}$, E. Alper Yıldırım ${ }^{\mathrm{b}, 1}$ \\ a Department of Industrial Engineering, Bilkent University, 06800 Bilkent, Ankara, Turkey \\ b Department of Industrial Engineering, Koç University, Rumelifeneri Yolu, 34450 Sariyer, İstanbul, Turkey
}

\section{A R T I C L E I N F O}

Available online 8 January 2013

Keywords:

Wireless mesh networks

Mixed integer linear programming

Heuristic method

Valid inequality

\begin{abstract}
A B S T R A C T
Wireless mesh networks (WMNs) provide cost effective solutions for setting up a communications network over a certain geographic area. In this paper, we study strategic problems of WMNs such as selecting the gateway nodes along with several operational problems such as routing, power control, and transmission slot assignment. Under the assumptions of the physical interference model and the tree-based routing restriction for traffic flow, a mixed integer linear programming (MILP) formulation is presented, in which the objective is to maximize the minimum service level provided at the nodes. A set of valid inequalities is derived and added to the model in an attempt to improve the solution quality. Since the MILP formulation becomes computationally infeasible for larger instances, we propose a heuristic method that is aimed at solving the problem in two stages. In the first stage, we devise a simple MILP problem that is concerned only with the selection of gateway nodes. In the second stage, the MILP problem in the original formulation is solved by fixing the gateway nodes from the first stage. Computational experiments are provided to evaluate the proposed models and the heuristic method. (c) 2013 Elsevier Ltd. All rights reserved.
\end{abstract}

\section{Introduction}

Wireless mesh networks (WMNs) are communication networks that consist of radio nodes that are organized in a mesh topology. WMNs emerged as low-cost alternatives for the "last mile" Internet access in developing areas, as they require wired connections to only a small number of their nodes, called gateways. Customer traffic at other nodes is carried over to these gateway nodes using the wireless media in a multihop fashion to access the Internet. In contrast to ad hoc networks, WMNs consist of stationary nodes with no energy conservation concerns, which aggregate the traffic of many customers in their coverage area. Therefore, their traffic inputs to the network have small variations over time.

In wireless communications, simultaneous transmission over the same frequency band from different nodes may cause interference at the receiving ends depending on the locations of the

\footnotetext{
This work was supported in part by TÜBITTAK (Turkish Scientific and Technological Research Council) Grant 110M312.

* Corresponding author. Tel.: +90312 2903343.

E-mail addresses: kgokbayr@bilkent.edu.tr (K. Gokbayrak), alperyildirim@ku.edu.tr (E.A. Yıldırım).

${ }^{1}$ This author was supported in part by TÜBITAK (Turkish Scientific and Technological Research Council) Grant 109M149 and by TÜBA-GEBIP (Turkish Academy of Sciences Young Scientists Award Program).
}

transmitting and the receiving nodes. Gupta and Kumar [8] suggested two alternative ways to model the impact of interference. In their protocol interference model, an "interference region" is defined for each node. Wireless communication from node $A$ to node $B$ is successful only if there is no other transmitting node $C$ whose interference region contains node $B$. The protocol interference model provides a simple approximation scheme for the interference. On the other hand, their physical interference model is more realistic as it reflects the additive nature of the interferences from all transmitting nodes: The communication from node $A$ to node $B$ is successful if the signal to interference and noise ratio (SINR) at node $B$ exceeds a certain threshold, which depends on the data rate. In this study, we assume that all links have the same data rate and therefore employ the physical interference model with a single SINR threshold value. Note that several transmissions can occur simultaneously if their SINR values exceed this threshold, and these transmissions are called non-interfering transmissions.

In this paper, we assume that the method of choice for dealing with interference is OFDMA (orthogonal frequency division multiple access, see [7]), an increasingly popular modulation method. In OFDMA, different transmissions use different time intervals and frequency channels or bands, so they theoretically do not interfere with each other. Fig. 1 illustrates an example with eight time intervals and six frequency bands, where each of the four colors represents a different set of non-interfering transmissions 


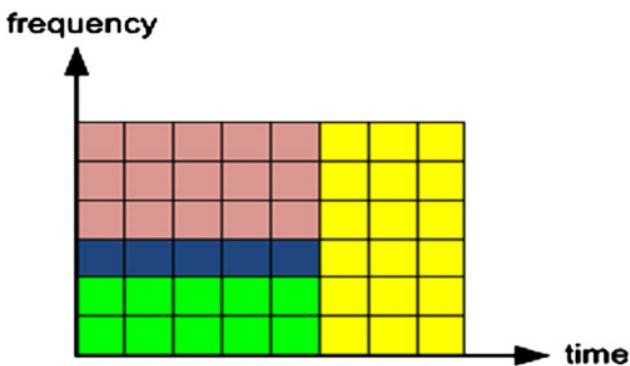

Fig. 1. OFDMA scheme.

that are active in the corresponding transmission slot. Note that the same scheme repeats in time.

According to the physical interference model, increasing the transmission power at a node will improve the SINR of its signal received at the other nodes, which may possibly enable new connections. This increase in power, however, will cause additional interference, possibly disabling some simultaneous connections at the same frequency band between other pairs of nodes. Hence, power control provides flexibility in forming noninterfering sets and this study considers this feature.

We assume that the locations and the maximum transmission power levels of the nodes are given so that all possible links of the WMN can be determined. For each node, there is a fixed rate of incoming traffic coming from the clients in the vicinity of that node. We are interested in carrying the traffic of each node in a multihop fashion to the Internet through the gateway nodes. Our objective is, for fairness, to provide capacities to nodes in proportion to their demands, and maximize the service level, defined as the minimum ratio of the assigned capacity to the node's demand over all nodes. For this task, we first propose a mixed integer linear programming (MILP) model: (i) to select a given number of gateway nodes, (ii) to determine the transmission power levels, (iii) to determine the routing of traffic from all nodes to gateways, and (iv) to determine sets of non-interfering links that can be assigned to the same transmission slot of the OFDMA scheme. In an attempt to improve the solution quality of the resulting optimization model, we derive a set of valid inequalities.

Even with the addition of the valid inequalities, the MILP formulation is difficult to solve to optimality especially for large instances. Therefore, we propose a heuristic method that is aimed at solving the problem in two stages. In the first stage, we devise a simple and effective heuristic method that is concerned only with the selection of gateway nodes. In the second stage, a simpler MILP problem is solved by fixing the gateway nodes from the first stage in the original formulation.

The paper is organized as follows. We discuss the related studies in the literature in Section 2. We propose a mixed integer linear programming formulation of the problem, present several bounds on its optimal value, introduce a set of valid inequalities, and propose a heuristic approach for gateway selection in Section 3. We propose a formal design process in Section 4 and Section 5 presents the computational results. Finally, we conclude the paper with some remarks in Section 6.

\section{Related work}

Over the last decade, there has been an extensive research effort on WMNs. We can only name a few of them in the discussion below. For other references, the reader is referred to survey papers, e.g., [1] and [12].

The effects of interference on wireless networks were first studied by Gupta and Kumar [8]. Defining throughput as the time average of the number of bits per second that can be transmitted by every node to its destination, they showed that the throughput capacity per node is $\Theta(1 / \sqrt{n \log n})$ in a random wireless network consisting of $n$ identical nodes that are communicating arbitrarily. When the locations of the nodes and the communications are optimized, the throughput capacity per node increases as $\Theta(1 / \sqrt{n})$. For WMNs where all nodes send or receive their traffic to or from the gateways, throughput capacity per node decreases as $\Theta(1 / n)$, as shown in [10], due to congestion at the gateways.

In order to alleviate the interference effect, two methods are proposed in the literature: channel assignment and link scheduling. Raniwala et al. [13] showed that the channel assignment to nodes is an NP-hard problem. Alicherry et al. [2] and Kodialam and Nandagopal [11] presented MILP formulations that employ the protocol interference model and multipath routing to address the joint channel assignment and routing problem with different objectives. The former has the same per-node-throughput fairness objective as in our paper, while the latter searches for a feasible solution that meets the node demands. The link scheduling problem for throughput maximization is shown to be NP-hard by Jain et al. [9], even with the simpler protocol interference model. Badia et al. [3] formulated an MILP problem for throughput maximization under the physical interference model and proposed a genetic algorithm for its solution.

Behzad and Rubin [4] considered a network with predetermined routing and developed an MILP formulation to minimize the schedule length under link scheduling and power control. They assumed the physical interference model and showed that this problem is NP-hard. Capone et al. [6] added the routing decisions and the rate adaptation to this MILP formulation and proposed a two-phase solution approach based on the column generation technique: Sets of non interfering links (configurations) are selected and then these configurations are assigned to transmission slots. Targon et al. [14], on the other hand, presented an MILP formulation for minimizing the gateway placement costs under routing and link scheduling. Two alternative interference constraints were given for both the protocol and the physical interference models. Similarly, Caillouet et al. [5] presented a generic MILP formulation to address various objectives under gateway placement, routing, and scheduling.

Our work differs from previous studies in several aspects: First, we assume that traffic flows are indivisible and consider a treestructured single-path routing, which eliminates the requirement of source-based routing capabilities on the routers. In our MILP model, the packets are routed independently of their sources. Second, we employ a power control scheme at the nodes so as to improve the spatial reuse. However, for each node, our model determines a single power level for transmission and uses that level at each transmission slot in which that node transmits. This approach is more realistic since the use of different power levels at different transmission slots does not seem to be feasible in practice. Finally, we tackle the problems of gateway placement, routing, transmission slot assignment, and power control jointly under the physical interference model. To the best of our knowledge, our study is the first one that addresses all of these problems simultaneously. The rate adaptation problem is left for future work.

In this study, we assume that the number of gateways and the demand of each node are given. Our objective is to provide capacities to nodes in proportion to their demands for fairness, and maximize the capacity utilization of the network.

\section{Problem formulation}

We consider the uplink traffic of a WMN composed of $N$ nodes, where node $i$ transmits at a maximum power of $P_{i}^{\max }$ to deliver its 
node demand $d_{i}, i=1, \ldots, N$. Due to the path loss $l_{i j} \in(0,1)$ between nodes $i$ and $j$, that transmission is received at node $j$ with the maximum signal strength of $g_{i j}=P_{i}^{\max } l_{i j}$.

We assume that a wireless link with data rate $c$ exists between nodes $i$ and $j$ if the signal-to-interference-plus-noise ratio (SINR) at node $j$ exceeds a threshold $\theta$ under the maximum transmission power $P_{i}^{\max }$ given that no other nodes are transmitting. Hence, the set of edges (links) of this network can be defined as

$E=\left\{(i, j) \mid g_{i j} \geq \theta \gamma_{j}\right\}$,

where $\gamma_{j}$ is the thermal noise strength at node $j$.

Utilizing OFDMA, we assume that there are $T$ time intervals and $K$ orthogonal frequency bands (channels) in a frame, hence the wireless capacity is divided into TK equal-data-rate transmission slots. We assume that the same frame structure is repeated periodically.

We define the ratio of transmission power of node $i$ at time interval $t$ on channel $k$ to $P_{i}^{\max }$ as $P_{i}^{t k} \in[0,1]$, and note that, according to the physical interference model, node $i$ can transmit to node $j$ successfully in this transmission slot $(t, k)$ if the SINR exceeds the threshold $\theta$, i.e.,

$\frac{P_{i}^{t k} g_{i j}}{\gamma_{j}+\sum_{\substack{n=1 \\ n \neq i, j}}^{N} P_{n}^{t k} g_{n j}} \geq \theta$.

On the left-hand side of the expression in (1), the numerator corresponds to the signal strength of node $i$ received at node $j$, whereas the denominator is given by the summation of the thermal noise at node $j$ and the total signal strength received by node $j$ from other transmitting nodes. For practical purposes, we assume that $P_{i}^{t k}$ can take values only from the set $\left\{0, \pi_{i}\right\}$, where $\pi_{i} \in[0,1]$ is also a decision variable that corresponds to power control at node $i$.

We assume that we have a budget for $G$ identical gateways, which have to be selected from among the WMN nodes. Each gateway will be installed a wired link of data rate $a$ connecting to the Internet. We also assume the half-duplex operation of nodes, i.e., a node cannot both transmit and receive in the same transmission slot.

In addition to $P_{i}^{t k}$ and $\pi_{i}$, the following decision variables are employed in the formulation:

$f_{i j}$ : The reserved capacity for traffic flow on link $(i, j),(i, j) \in E$.

$w$ : The ratio of the allocated input capacity at nodes to the demand amount.

$x_{i j}^{t k}= \begin{cases}1 & \text { if wireless link }(i, j) \text { is active at transmission slot }(t, k), \\ 0 & \text { otherwise, }\end{cases}$

$(i, j) \in E, \quad t \in\{1, \ldots, T\}, \quad k \in\{1, \ldots, K\}$.

$z_{i j}=\left\{\begin{array}{ll}1 & \text { if wireless link }(i, j) \text { is ever active, } \\ 0 & \text { otherwise, }\end{array}(i, j) \in E\right.$.

$y_{i}=\left\{\begin{array}{ll}1 & \text { if node } i \text { is a gateway, } \\ 0 & \text { otherwise, }\end{array} i \in\{1, \ldots, N\}\right.$.

We define the MILP, denoted by (WMN1), as

$\max w$

subject to

(i) Flow conservation and gateway capacity constraints

$$
\begin{aligned}
& w d_{i}+\sum_{s:(s, i) \in E} f_{s i} \leq a y_{i}+\sum_{j:(i, j) \in E} f_{i j}, \quad i \in\{1, \ldots, N\}, \\
& w d_{i}+\sum_{s:(s, i) \in E} f_{s i} \geq \sum_{j:(i, j) \in E} f_{i j}, \quad i \in\{1, \ldots, N\} .
\end{aligned}
$$

(ii) Wireless link capacity constraints

$f_{i j} \leq \frac{c}{T K} \sum_{t=1}^{T} \sum_{k=1}^{K} x_{i j}^{t k},(i, j) \in E$

(iii) Single path routing constraints

$\sum_{j:(i, j) \in E} z_{i j}=1-y_{i}, \quad i \in\{1, \ldots, N\}$.

(iv) Link activation constraints

$x_{i j}^{t k} \leq z_{i j},(i, j) \in E, \quad t \in\{1, \ldots, T\}, \quad k \in\{1, \ldots, K\}$.

(v) Half-duplex communication constraints

$\sum_{s:(s, i) \in E} x_{s i}^{t k}+\sum_{j:(i, j) \in E} x_{i j}^{t k} \leq 1, \quad i \in\{1, \ldots, N\}, \quad t \in\{1, \ldots, T\}, \quad k \in\{1, \ldots, K\}$.

(vi) Power schedule constraints

$P_{i}^{t k} \leq \sum_{j:(i, j) \in E} x_{i j}^{t k}, \quad i \in\{1, \ldots, N\}, \quad t \in\{1, \ldots, T\}, \quad k \in\{1, \ldots, K\}$,

$P_{i}^{t k} \leq \pi_{i}, \quad i \in\{1, \ldots, N\}, \quad t \in\{1, \ldots, T\}, \quad k \in\{1, \ldots, K\}$,

$P_{i}^{t k} \geq \pi_{i}+\sum_{j:(i, j) \in E} x_{i j}^{t k}-1, \quad i \in\{1, \ldots, N\}, \quad t \in\{1, \ldots, T\}, \quad k \in\{1, \ldots, K\}$.

(vii) Physical interference constraints

$P_{i}^{t k} g_{i j}+M_{i j}\left(1-x_{i j}^{t k}\right) \geq \theta \gamma_{j}+\theta \sum_{\substack{n=1 \\ n \neq i j}}^{N} P_{n}^{t k} g_{n j}, \quad(i, j) \in E, \quad t \in\{1, \ldots, T\}, \quad k \in\{1, \ldots, K\}$,

where

$M_{i j}=\theta\left(\gamma_{j}+\sum_{\substack{n=1 \\ \varepsilon \neq 1, j}}^{N} g_{n j}\right), \quad(i, j) \in E$

(viii) Gateway budget constraint

$\sum_{i=1}^{N} y_{i}=G$

(ix) Binary variables

$x_{i j}^{t k} \in\{0,1\}, \quad(i, j) \in E, \quad t \in\{1, \ldots, T\}, \quad k \in\{1, \ldots, K\}$,

$y_{i} \in\{0,1\}, \quad i \in\{1, \ldots, N\}$,

$z_{i j} \in\{0,1\}, \quad(i, j) \in E$.

(x) Non-negative real variables

$P_{i}^{t k} \geq 0, \quad i \in\{1, \ldots, N\}, \quad t \in\{1, \ldots, T\}, \quad k \in\{1, \ldots, K\}$,

$\pi_{i} \geq 0, \quad i \in\{1, \ldots, N\}$,

$f_{i j} \geq 0, \quad(i, j) \in E$,

$w \geq 0$.

Our objective in (2) is to maximize the service level $w$. Assuming that the traffic from nodes will increase proportionally over time, this objective serves to design the network that can last the longest without any upgrades. Note that, in order to meet the current demand of each node, the service level has to exceed one, but finding the number of gateways $G$ to meet the demand is not the subject of study in this paper. We remark, however, that our formulation can still be used for this purpose by simply performing a binary search on the number of gateways $G$. Inequalities (3) and (4) are the flow balance constraints that also incorporate 
the wired link capacities at the gateways. The capacities of the wireless links are proportional to the number of transmission slots at which they are active, and these capacities are translated into upper bounds on the flows as in (5). For each node that is not a gateway, Eq. (6) ensures a single outgoing link. Note that this constraint alone does not necessarily guarantee a tree structure, as loops are possible. However, in case of loops, no traffic can be routed from the nodes in the loop to a gateway, which results in a service level of zero. Inequality (7) prevents the links that are not on any route from being activated in any transmission slot. Nodes cannot transmit and receive in the same slot as in the half-duplex communication constraints (8). Inequalities (9)-(11) are included to linearize the constraint

$P_{i}^{t k}=\pi_{i} \sum_{j:(i, j) \in E} x_{i j}^{t k}$,

i.e., when an outgoing link of node $i$ is activated at the transmission slot $t k$, the power ratio $P_{i}^{t k}$ is set to $\pi_{i}$, otherwise it is set to zero. (It follows from (8) that $\sum_{j:(i, j) \in E} x_{i j}^{t k}$ is either zero or one.) The successful transmission under the physical interference model (1) is represented in (12) in the form of a big-M constraint, and the smallest possible value that the parameter $M$ can take is provided. The number of gateways is given in (13), and all the binary and the continuous decision variables are specified in (14)-(20).

(WMN1) is an MILP problem that consists of $5 N T K+2|E| T K+$ $4 N+2|E|+2$ constraints, $N T K+N+|E|+1$ continuous variables, and $|E| T K+|E|+N$ binary variables. Therefore, the size of the optimization problem grows quickly as the number of nodes $N$, the number of edges $|E|$, the number of time slots $T$, and the number of channels $K$ increase.

In the following two sections, we derive upper bounds on the optimal value of (WMN1) and present a set of valid inequalities in an attempt to improve the solution quality of (WMN1), respectively.

\subsection{Upper bounds for the optimal value}

In this section, we determine upper bounds for the optimal value of the optimization model (WMN1). To start out, we establish a closed-form expression of the optimal value of the LP relaxation of (WMN1) by constructing an optimal solution.

Lemma 1. Suppose that $c \leq a$ in the optimization model (WMN1). The optimal value of the $L P$ relaxation of (WMN1) is given by

$\hat{w}:=\min \left(\frac{G a}{\sum_{i=1}^{N} d_{i}}, \frac{a}{\max _{i \in\{1,2, \ldots, N\}} d_{i}}\right)$.

It follows that:

$w^{*} \leq \hat{w}$,

where $w^{*}$ denotes the optimal value of (WMN1).

Proof. Note that the summation of the inequalities (3) over all nodes together with (13) yields

$w \leq \frac{G a}{\sum_{i=1}^{N} d_{i}}$,

for any feasible solution of the LP relaxation of (WMN1). Similarly, for each node $i \in\{1, \ldots, N\}$, we have

$$
\begin{aligned}
w d_{i} & \leq a y_{i}+\sum_{j:(i, j) \in E} f_{i j}, \\
& \leq a y_{i}+\frac{c}{T K} \sum_{j:(i, j) \in E} \sum_{t=1}^{T} \sum_{k=1}^{K} x_{i j}^{t k}, \\
& \leq a y_{i}+\frac{c}{T K} \sum_{j:(i, j) \in E} \sum_{t=1}^{T} \sum_{k=1}^{K} z_{i j},
\end{aligned}
$$

$$
\begin{aligned}
& =a y_{i}+c \sum_{j:(i, j) \in E} z_{i j}, \\
& =a y_{i}+c\left(1-y_{i}\right), \\
& \leq a,
\end{aligned}
$$

where we used the non-negativity of $f_{i j}$ for each $(i, j) \in E$ and $(3)$ in the first line, (5) in the second line, (7) in the third line, (6) in the fifth line, and the assumption that $c \leq a$ together with $y_{i} \in[0,1]$ in the LP relaxation of (WMN1) in the last line. It follows that $w \leq a / d_{i}$ for each node $i$ such that $d_{i}>0$. Therefore, for any feasible solution of the LP relaxation of (WMN1), we have

$w \leq \frac{a}{\max _{i \in\{1,2, \ldots, N\}} d_{i}}$

We prove (21) by constructing a feasible solution of the LP relaxation of (WMN1) whose objective function value attains the smaller of the two upper bounds (23) and (24). Assume first that (23) is smaller than or equal to (24), i.e.,

$$
G \leq \frac{\sum_{i=1}^{N} d_{i}}{\max _{i \in\{1,2, \ldots, N\}} d_{i}}
$$

In this case, the idea is to treat each node as a fractional gateway and to have each node transfer its traffic directly to the Internet. Let us define $y_{j}=\left(G d_{j}\right) /\left(\sum_{i=1}^{N} d_{i}\right)$ for each $j \in\{1,2, \ldots, N\}$. Note that $y_{j} \in[0,1]$ for each node $j$ by (25). We define $f_{i j}=0, P_{i}^{t k}=0, x_{i j}^{t k}=0$, and $\pi_{i}=0$ for each possible choice of the indices. For $z_{i j}$, we choose any non-negative solution that satisfies (6). Setting $w$ equal to the upper bound (23), it is easy to verify that the corresponding solution satisfies all the constraints of the LP relaxation of (WMN1). Suppose now that (23) is larger than (24), i.e.,

$$
G>\frac{\sum_{i=1}^{N} d_{i}}{\max _{i \in\{1,2, \ldots, N\}} d_{i}}
$$

In this case, the solution constructed in the first case is no longer feasible for the LP relaxation of (WMN1) since $y_{j}>1$ for nodes $j$ with demand values $d_{j}>\left(\sum_{i=1}^{N} d_{i}\right) / G$. Let $j^{*}$ denote the index of a node with the largest demand. Note that there may be multiple nodes with such demand. We now scale the previously computed $y_{j}$ values by multiplying each one by $\left(1 / y_{j^{*}}\right)$ so that the largest one is equal to one. Since the constraint (13) is now violated, we apply the following feasibility restoration scheme: We arbitrarily increase the $y_{j}$ value of each node $j$ whose scaled $y_{j}$ value is strictly less than one, without exceeding one, and thereby ensuring that (13) is again satisfied. Note that this is always possible since $G \leq N$. We next set $w$ equal to (24). The remaining components of the solution are defined exactly as in the first case. It follows that the resulting solution is feasible for the LP relaxation of (WMN1), which completes the proof.

Lemma 1 yields an upper bound on $w^{*}$. It is worth noticing that the optimal value $\hat{w}$ of the LP relaxation is linearly increasing with the number of gateways $G$ up to $L\left(\sum_{i=1}^{N} d_{i}\right) /\left(\max _{i \in\{1,2, \ldots, N\}}\right.$ $\left.\left.d_{i}\right)\right\rfloor$, and remains constant when the number of gateways exceeds this threshold.

Each node has a maximum input capacity of $c$ for traffic originating from other nodes, which can be realized when one of the incoming links is active at all transmission slots. Since the gateways are not sending their traffic to other gateways, the total amount of traffic reaching the gateways on their wireless links is the total demand of the non-gateway nodes. When the number of gateways is $G$, defining $D_{G}$ as the sum of $G$ highest node demands, 
another upper bound for $w^{*}$ can be given as

$w^{*} \leq \frac{G c}{\sum_{i=1}^{N} d_{i}-D_{G}}$.

In practice, the gateway capacities $a$ are much larger than the wireless link capacities $c$. Therefore, the upper bound in (27) will be tighter than the LP relaxation bound in (21) when the number of gateways $G$ is sufficiently small, i.e., when $G$ satisfies both of the inequalities

$\frac{(a-c)}{a} \sum_{i=1}^{N} d_{i} \geq D_{G}$

and

$\sum_{i=1}^{N} d_{i} \geq D_{G}+\frac{c G}{a} \max _{i \in\{1, \ldots, N\}} d_{i}$.

\subsection{Valid inequalities}

In this section, we present a set of valid inequalities that prevents certain pairs of links from being activated simultaneously in the same transmission slot by properly exploiting a sufficient condition.

Proposition 2. Given an instance of (WMN1), for each link $(i, j) \in E$, let us define its conflict set as

$$
\begin{aligned}
C_{(i, j)}: & =\left\{(s, d) \in E, s \neq i, s \neq j, d \neq i, d \neq j: g_{i j} g_{s d}-\theta^{2} g_{i d} g_{s j}\right. \\
& \left.<\theta \max \left(g_{s d} \gamma_{j}+\theta g_{s j} \gamma_{d}, g_{i j} \gamma_{d}+\theta g_{i d} \gamma_{j}\right)\right\} .
\end{aligned}
$$

The following set of inequalities is valid for (WMN1):

$x_{i j}^{t k}+x_{s d}^{t k} \leq 1, \quad(i, j) \in E, \quad(s, d) \in C_{(i, j)}, \quad t \in\{1, \ldots, T\}, \quad k \in\{1, \ldots, K\}$.

Proof. We prove the contrapositive of the statement. For a given $t \in\{1, \ldots, T\}$ and $k \in\{1, \ldots, K\}$, suppose that there exist two links $(i, j) \in E$ and $(s, d) \in E$ that are simultaneously active in the same transmission slot, i.e., $x_{i j}^{t k}=x_{s d}^{t k}=1$. We will show that $(s, d) \notin C_{(i, j)}$. By (12), we have

$P_{i}^{t k} g_{i j} \geq \theta \gamma_{j}+\theta P_{s}^{t k} g_{s j}+\theta \sum_{\substack{n=1 \\ n \neq i, j, s}}^{N} P_{n}^{t k} g_{n j}$

and

$P_{s}^{t k} g_{s d} \geq \theta \gamma_{d}+\theta P_{i}^{t k} g_{i d}+\theta \sum_{\substack{n=1 \\ n \neq i, s, d}}^{N} P_{n}^{t k} g_{n d}$

Multiplying (30) by $g_{s d}$ and substituting (31), we obtain

$P_{i}^{t k}\left(g_{i j} g_{s d}-\theta^{2} g_{i d} g_{s j}\right) \geq \theta\left(g_{s d} \gamma_{j}+\theta g_{s j} \gamma_{d}\right)+\theta^{2} \sum_{\substack{n=1 \\ n \neq i, s, d}}^{N} P_{n}^{t k} g_{s j} g_{n d}+\theta \sum_{\substack{n=1 \\ n \neq i, j, s}}^{N} P_{n}^{t k} g_{s d} g_{n j}$.

Since $P_{i}^{t k}>0$ and the right-hand side is positive, it follows that:

$g_{i j} g_{s d}-\theta^{2} g_{i d} g_{s j}>0$

and since $P_{i}^{t k} \leq 1$, we obtain

$\left(g_{i j} g_{s d}-\theta^{2} g_{i d} g_{s j}\right) \geq \theta\left(g_{s d} \gamma_{j}+\theta g_{s j} \gamma_{d}\right)+\theta^{2} \sum_{\substack{n=1 \\ n \neq i, s, d}}^{N} P_{n}^{t k} g_{s j} g_{n d}+\theta \sum_{\substack{n=1 \\ n \neq i, j, s}}^{N} P_{n}^{t k} g_{s d} g_{n j}$.
In a similar fashion, the following condition can be derived by multiplying (31) by $g_{i j}$ and substituting (30):

$\left(g_{i j} g_{s d}-\theta^{2} g_{i d} g_{s j}\right) \geq \theta\left(g_{i j} \gamma_{d}+\theta g_{i d} \gamma_{j}\right)+\theta^{2} \sum_{\substack{n=1 \\ n \neq s, i, j}}^{N} P_{n}^{t k} g_{i d} g_{n j}+\theta \sum_{\substack{n=1 \\ n \neq s, d, i}}^{N} P_{n}^{t k} g_{i j} g_{n d}$.

By the non-negativity of the second and the third terms on the right-hand sides of (32) and (33), we obtain

$\left(g_{i j} g_{s d}-\theta^{2} g_{i d} g_{s j}\right) \geq \theta \max \left(g_{s d} \gamma_{j}+\theta g_{s j} \gamma_{d}, g_{i j} \gamma_{d}+\theta g_{i d} \gamma_{j}\right)$

which implies that $(s, d) \notin C_{(i, j)}$ due to $(28)$.

Note that if link $(s, d) \in C_{(i, j)}$, then link $(i, j) \in C_{(s, d)}$, therefore half of the inequalities (29) can be eliminated from the formulation.

\section{Proposed design process}

As we will demonstrate with our computational experiments, (WMN1) is a very hard problem to solve to optimality even for small networks. Hence, we propose the following approach to obtain near-optimal results.

We first separate the gateway selection problem from the rest. Once the gateway locations are determined, they can be fixed in (WMN1), which leads to a simpler optimization problem in comparison with the original formulation. To determine the gateway locations, we employ the following heuristic method: We consider an uncapacitated wired network among the nodes so that wireless interference, power control, and transmission slot assignments are ignored. We attempt to route the traffic of each node through a prespecified number of gateways. Our objective is to locate the gateways so as to minimize the total traffic flow on this network:

$\min \sum_{(s, d) \in E} f_{s d}$

subject to the constraints (6), (13), (15), (16), (19), and

$d_{i}+\sum_{s:(s, i) \in E} f_{s i}-\sum_{d:(i, d) \in E} f_{i d} \leq a y_{i}, \quad i \in\{1, \ldots, N\}$,

$d_{i}+\sum_{s:(s, i) \in E} f_{s i}-\sum_{d:(i, d) \in E} f_{i d} \geq 0, \quad i \in\{1, \ldots, N\}$,

$f_{i j} \leq c z_{i j}, \quad(i, j) \in E$,

$\sum_{s:(s, i) \in E} f_{s i}+\sum_{j:(i, j) \in E} f_{i j} \leq c, \quad i \in\{1, \ldots, N\}$.

We denote the resulting MILP formulation by (G1). The constraint (36), which suggests that traffic flow between nodes is allowed only on links included in a routing tree and is bounded above by the capacity of the wireless link, is obtained from (5) and (7). Similarly, the constraint (37) is obtained from (5) and (8) suggesting that the total input and output traffic flow on wireless links of a node is bounded above by the link capacity because it is possible to activate at most one link in a transmission slot.

The main motivation behind the minimization of the total flow on the links is to select the gateways in such a way that the amount of traffic that will be routed using the wireless connections will be relatively small. Once the gateway locations are determined, they will be fixed in (WMN1), which is then solved to determine the power control, routing, and transmission slot assignments.

Combining all these components together, we suggest the following two-step design process: Determine the gateway locations by solving (G1) and solve (WMN1) by fixing the selected 
gateways to determine the service level, routing, power control, and transmission schedule.

\section{Computational experiments}

In this section, we consider three networks whose topologies are given in Fig. 2a-c, where the node demands are presented in Mbps. Each gateway connection is assumed to have a capacity of $a=45 \mathrm{Mbps}$ and each link has a capacity of $c=24 \mathrm{Mbps}$ for the $\theta$ value of 50. Each node is assumed to have a maximum transmission power of $7.5 \mathrm{~mW}$ (measured at $1 \mathrm{~m}$ distance) and the thermal noise $\gamma$ is assumed to be $1.5 \times 10^{-10} \mathrm{~mW}$ at all nodes. The distance of each wireless link of the grid is $1 \mathrm{~km}$ and the path loss $l_{i j}$ is assumed to be inversely proportional to the cube of the distance between nodes $i$ and $j$.

We first study the behavior of the proposed upper bounds on the optimal service levels $w^{*}$. In particular, we plot the LP relaxation value in (21) and the upper bounds given by (27) as a function of the number of gateways $G$ on each network in

b
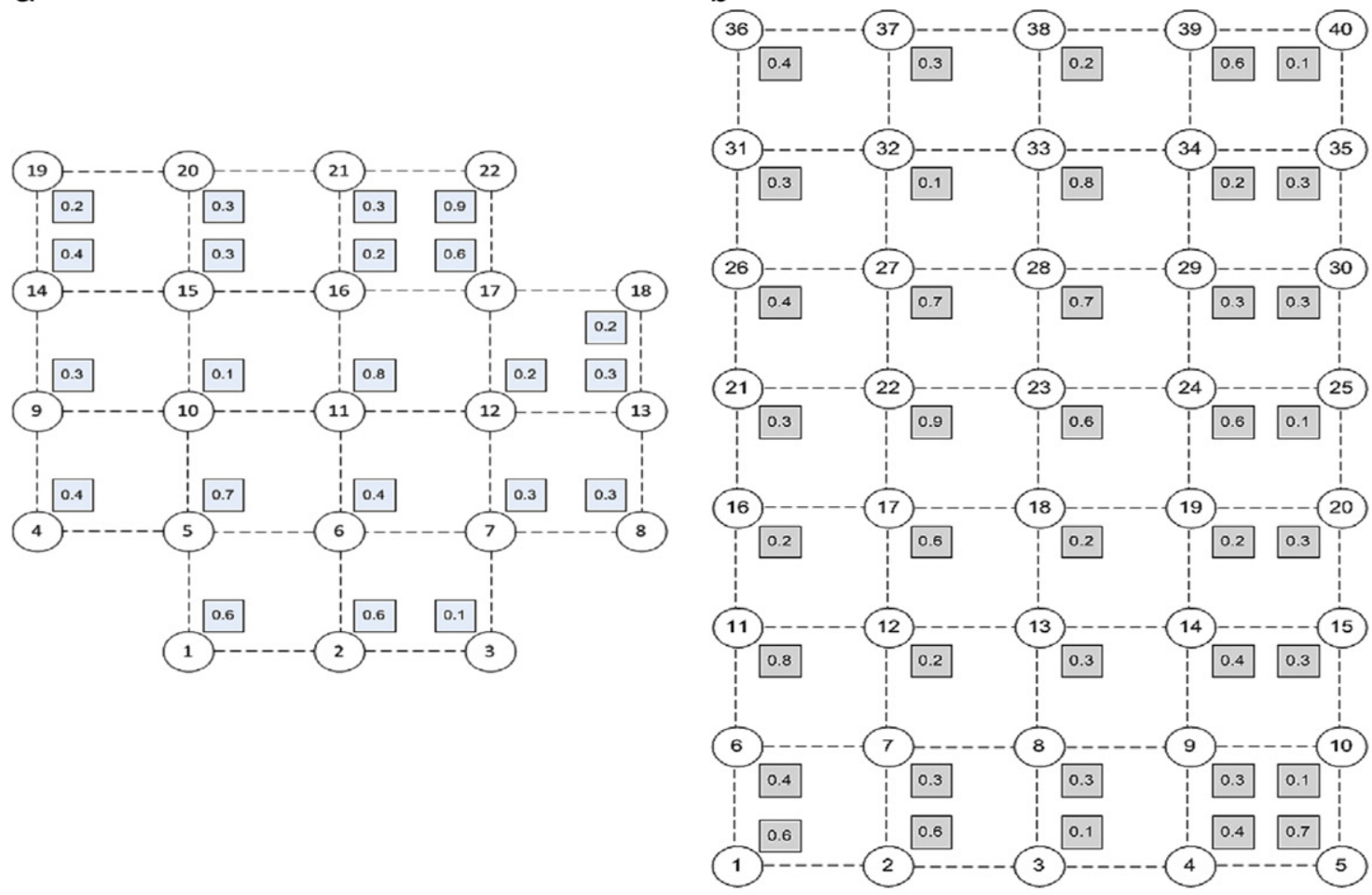

C

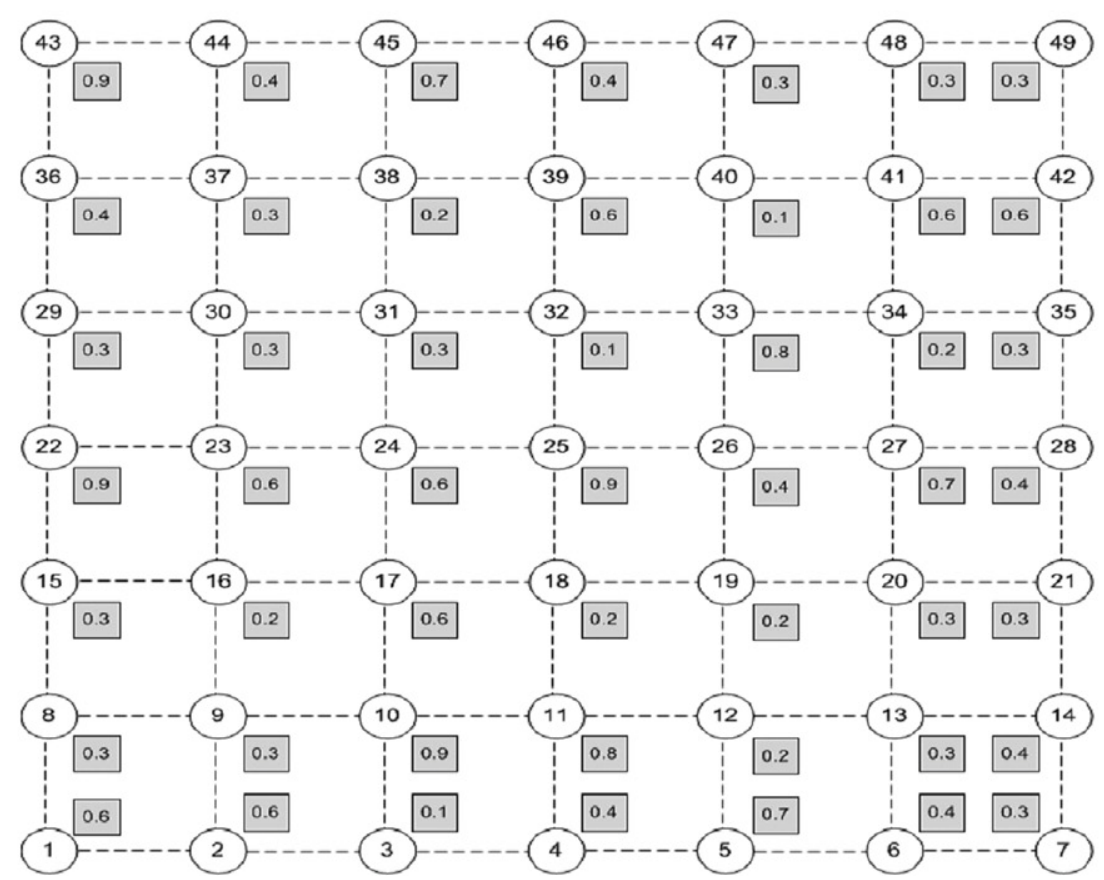

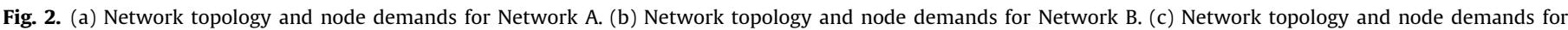
Network C. 
a

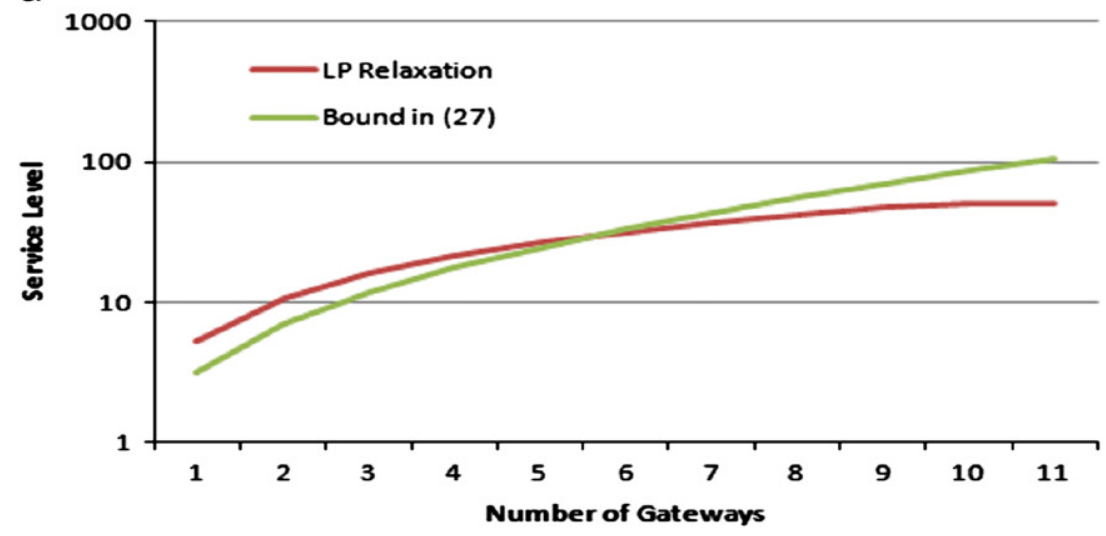

b

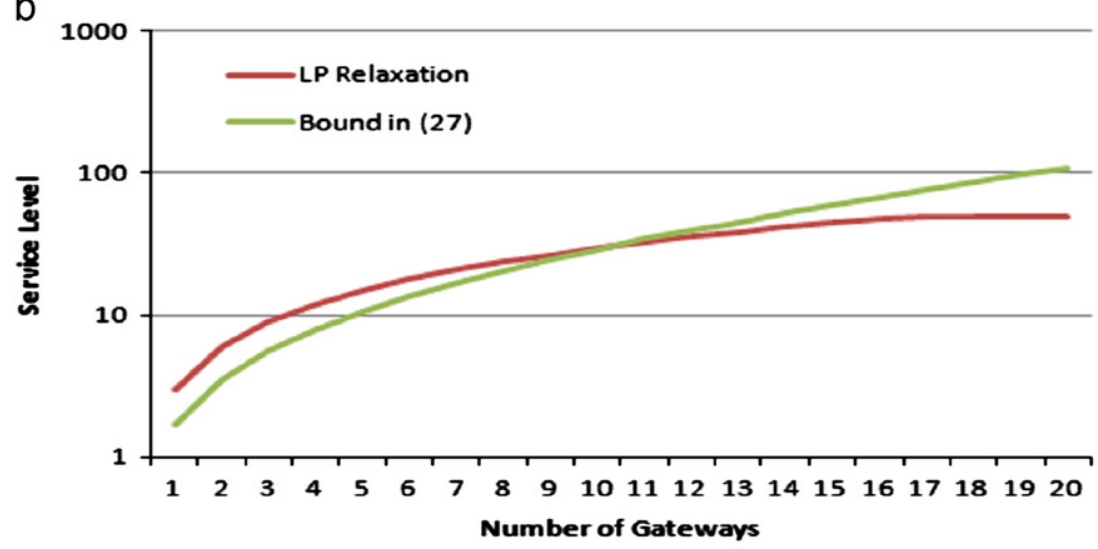

C

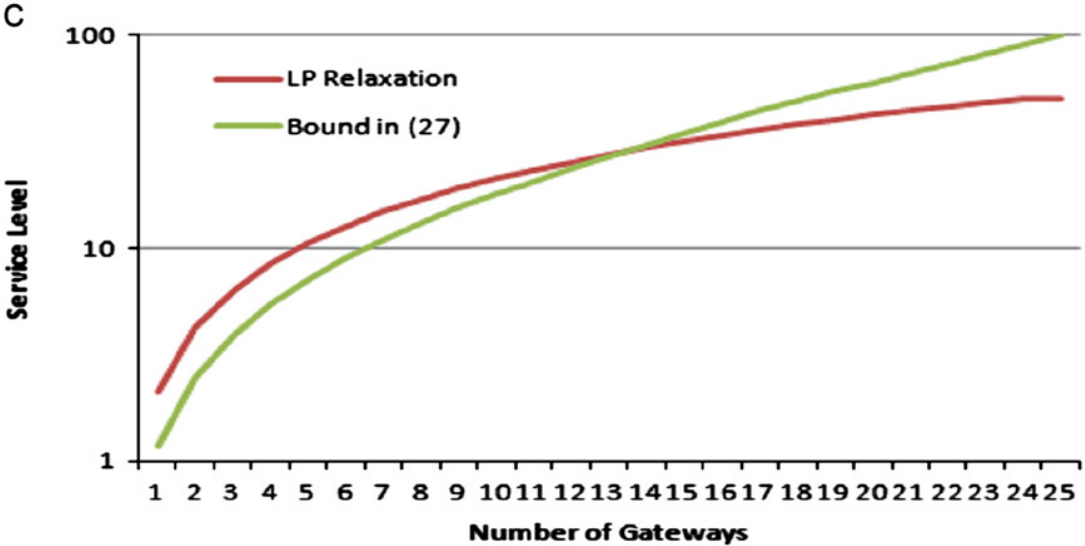

Fig. 3. (a) Bounds on the optimal value of Network A. (b) Bounds on the optimal value of Network B. (c) Bounds on the optimal value of Network C.

Fig. 3a-c. We remark that each upper bound depends on the number of gateways $G$ but is independent of the number of transmission slots TK and the interference threshold $\theta$. Note that we use a logarithmic scale for the service level.

As mentioned at the end of Section 3.1, Figs. 3a-c reveal that the upper bound given by (27) is tighter for smaller values of $G$, which corresponds to the range of interest.

We work with a single gateway in Network A, three gateways in Network B, and four gateways in Network C. In Table 1, we present the gateways obtained for the networks from the proposed heuristic. For comparison purposes, we also include a 'busiest router placement' heuristic (see [15]), which picks the $G$ highest traffic nodes as gateways. For simplicity, we use G1 and BRP to refer to each of these heuristic methods. Utilizing a laptop computer with a single Intel Core i7-740 CPU and 8 GB of memory
Table 1

Gateways selected by the heuristics.

\begin{tabular}{llrl}
\hline Heuristic & Network A & Network B & Network C \\
\hline G1 & 11 & $3,23,27$ & $11,22,34,45$ \\
BRP & 22 & $11,22,33$ & $10,22,25,43$ \\
\hline
\end{tabular}

running Gurobi 5.0.1, each of the optimization models (G1) was solved to optimality within a few minutes to obtain the gateways.

Table 1 reveals that each heuristic method may select a different subset of nodes as gateways. Note that the gateway selections are independent of the number of transmission slots TK and the SINR threshold $\theta$.

In order to compare G1 and BRP, we fixed the gateways in (WMN1) with the set of valid inequalities (29), and the solver was 
run with a limit of one hour on the same computer. For comparison purposes, we also solved them without prespecifying the gateways with the same time limit, which we denote by WMN1. The results along with the tightest upper bound given by (27) are presented in Fig. 4a-c. Note that since none of the instances could be solved to optimality, we present the best feasible solution and the best upper bound computed by the solver within the time limit. For each of the three networks, we constructed two instances using $T K \in\{32,64\}$ in our experiments.

For the simpler Network A, G1 found feasible solutions with performances better than those obtained using BRP. We remark that the service levels obtained from G1 were even better than the upper bounds on the optimal service levels that could be obtained using BRP. For this network, WMN1 returned feasible solutions with the same objective function values as those computed by G1. For the larger and more realistic sized Network B, WMN1 was unable to find a feasible solution for $T K=32$ and computed a feasible solution with a considerably large integrality gap for $T K=64$ within the one hour limit. G1 outperformed both WMN1 and BRP in terms of the quality of feasible solutions. Similarly, WMN1 could not compute a feasible solution within

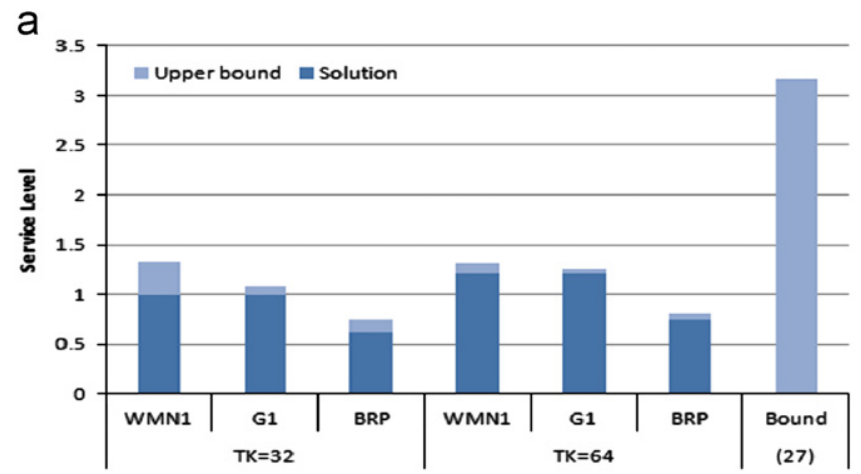

b

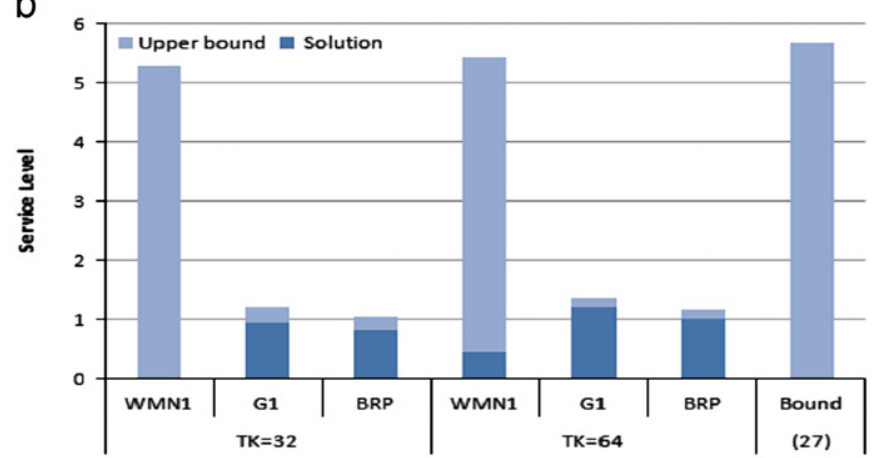

C

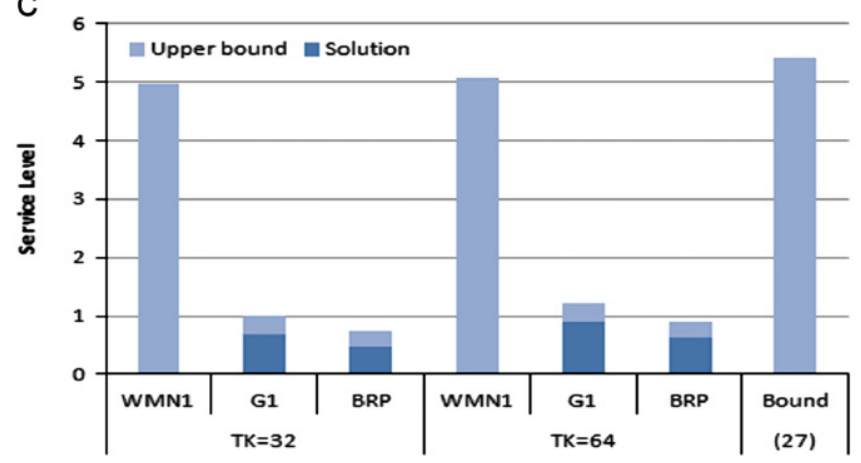

Fig. 4. (a) Network A solutions and the upper bound. (b) Network B solutions and the upper bound. (c) Network $C$ solutions and the upper bound. the time limit for both instances corresponding to the largest Network C. G1 outperformed BRP on both instances.

In Fig. 4a-c, we also included the upper bound (27). For the larger Networks B and C, the upper bound obtained using (WMN1) was only slightly better than the corresponding bounds (27), each of which is further smaller than the optimal value of the LP relaxation of (WMN1), as illustrated in Fig. 3b and c. This observation suggests that the integrality gap of the optimization model (WMN1) is fairly large and that the solver could not make significant improvements to the upper bound at the end of one hour, especially for larger instances. In contrast, the integrality gap of (G1) was fairly small at the end of one hour on all instances. In light of these observations and the computational evidence from our computational experiments, we suggest solving (G1) to determine the gateway locations and then solving (WMN1), possibly under a time limit, by fixing these gateway locations.

In order to illustrate the benefit of the valid inequalities, we compare the solutions of G1 with and without the valid inequalities in Fig. 5a-c. In the simpler network, Network A, the set of valid inequalities lowers the optimality gaps, while the best feasible solution performances are not affected. In the more realistic networks, Networks B and C, both the feasible solutions and the optimality gaps are improved. Therefore, the inclusion of this set of valid inequalities is recommended.

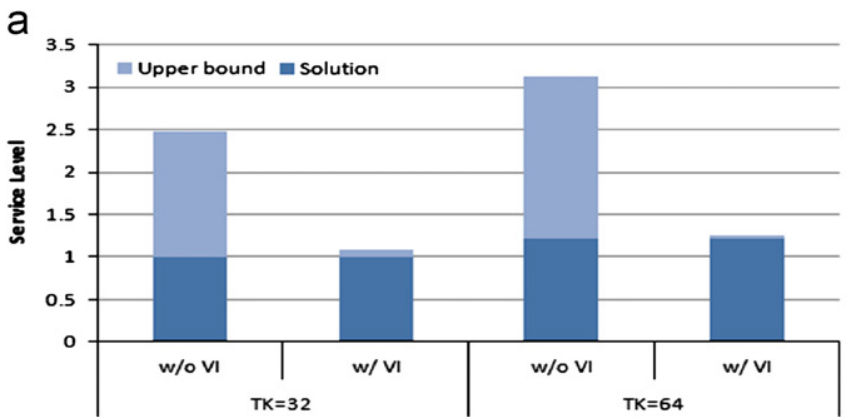

b

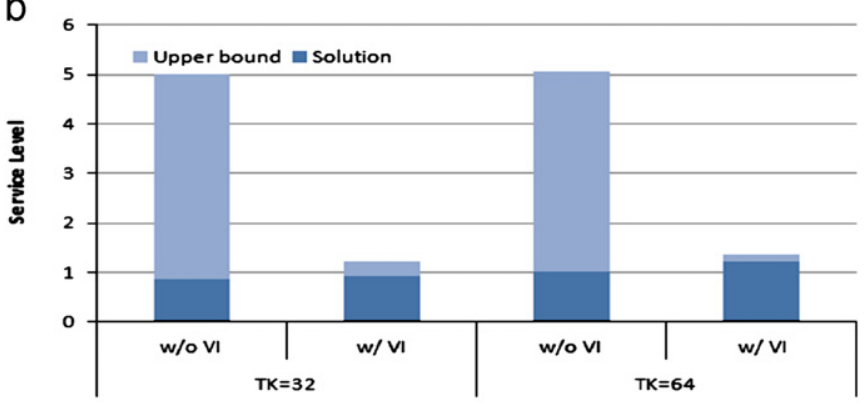

C

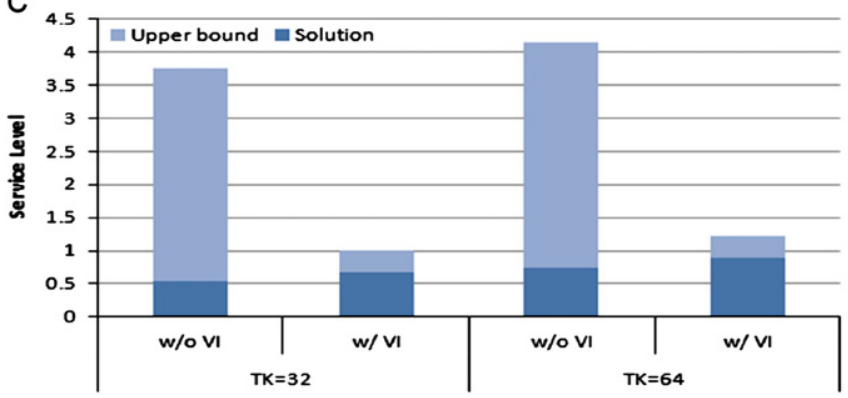

Fig. 5. (a) Benefit of the valid inequalities on Network A. (b) Benefit of the valid inequalities on Network B. (c) Benefit of the valid inequalities on Network C. 


\section{Conclusions and future work}

We presented an MILP formulation to optimize the gateway selection, transmission scheduling, power control, and routing in WMNs under the tree-based routing assumption. Our objective was to maximize the minimum ratio of the allocated capacity to each node's demand, which we defined as the service level. Assuming that node demands grow proportionally over time, this objective enabled us to design a network that can last the longest for a given number of gateway nodes.

Our experiments demonstrated that our MILP formulation may become computationally infeasible especially for larger instances of the problem. In that case, we proposed to solve the problem in two stages: In the first stage, we selected gateways by relaxing certain constraints of the original MILP formulation and modifying the objective function. Then, in the second stage, the resulting gateways were fixed in the original MILP model so that a simpler MILP problem was solved to determine the transmission schedule, power control, and routing. Our computational results illustrated that the resulting service levels were promising in terms of solution quality.

Our preliminary results suggest that replacing the tree-based routing assumption by the less restrictive source-based single path routing may lead to higher service levels for the same set of gateways. The complexity of the resulting optimization model, however, also increases with this modification, which restricts the applicability of the formulation to very small-size problems. We are currently investigating heuristic methods for this more difficult problem.

\section{Acknowledgments}

We are grateful to two anonymous referees whose comments and suggestions led to several improvements in the presentation.

\section{References}

[1] Akyıldız IF, Wang X, Wang W. Wireless mesh networks: a survey. Computer Networks 2005;47(4):445-87.

[2] Alicherry M, Bhatia R, Li LE. Joint channel assignment and routing for throughput optimization in multi-radio wireless mesh networks. In: Proceedings of the 11th annual international conference on mobile computing and networking; 2005. p. 58-72.

[3] Badia L, Botta A, Lenzini L. A genetic approach to joint routing and link scheduling for wireless mesh networks. Ad Hoc Networks 2009;7(4):654-64.

[4] Behzad A, Rubin I. Optimum integrated link scheduling and power control for multihop wireless networks. IEEE Transactions on Vehicular Technology 2007;56(1):194-205.

[5] Caillouet C, Perennes S, Rivano H. Framework for optimizing the capacity of wireless mesh networks. Computer Communications 2011;34:1645-59.

[6] Capone A, Carello G, Filippini I, Gualandi S, Malucelli F. Routing, scheduling and channel assignment in wireless mesh networks: optimization models and algorithms. Ad Hoc Networks 2010;8:545-63.

[7] Fazel K, Kaiser S. Multi-carrier and spread spectrum systems: from OFDM and MC-CDMA to LTE and WiMAX. Wiley; 2008.

[8] Gupta P, Kumar PR. The capacity of wireless networks. IEEE Transactions on Information Theory 2000;46(2):388-404.

[9] Jain K, Padhye J, Padmanabhan VN, Qiu L. Impact of interference on multi-hop wireless network performance. Wireless Networks 2005;11(4):471-87.

[10] Jun J, Sichitiu ML. The nominal capacity of wireless mesh networks. IEEE Wireless Communications 2003;10(5):8-14.

[11] Kodialam M, Nandagopal T. Characterizing the capacity region in multi-radio multi-channel wireless mesh networks. In: Proceedings of the 11th annual international conference on mobile computing and networking; 2005. p. 7387.

[12] Pathak PH, Dutta R. A survey of network design problems and joint design approaches in wireless mesh networks. IEEE Communications Surveys and Tutorials 2011;13(3):396-428.

[13] Raniwala A, Gopalan K, Chiueh T. Centralized channel assignment and routing algorithms for multi-channel wireless mesh networks. Mobile Computing and Communications Review 2004;8(2):50-65.

[14] Targon V, Sanso B, Capone A. The joint gateway placement and spatial reuse problem in wireless mesh networks. Computer Networks 2010;54(2): 231-40.

[15] Zhou P, Wang X, Manoj BS, Rao R. On optimizing gateway placement for throughput in wireless mesh networks. EURASIP Journal on Wireless Communications and Networking 2010;2010:1-12. 